\title{
Solar Cell Crack Identification using Internet of Things (IoT)
}

\author{
L. Nagalakshmi ${ }^{1}$, K.S. Abdul Karimullah Ansari ${ }^{2}$, Gowtham. ${ }^{3}$, Janagi Raman. ${ }^{4}$ \\ Assistant Professor, Department of Electrical and Electronics, S.A Engineering College ${ }^{1}$ \\ UG Student, Department of Electrical and Electronics, S.A Engineering College $e^{2,3,4}$
}

\begin{abstract}
The main aim of this project is to propose an automated inspection technique of solar cell panel to detect cracks and monitor its output round the clock. This monitoring is done from anywhere and anytime with the help of Internet of Things (IoT). From the manufacturer's point of view, the inspection of the solar cell panel is essentially performed to obtain a high quality product. The power generated by the cells can be simultaneously calculated, as the current value is updated periodically to the control room and/or to the mobile monitoring app via internet. When there is a drop in the current and voltage values of the solar panel, a notification is received in the developed application. Further a mail is being sent to the configured mail IDs regarding the parameters of the solar panel which in turn will help us to detect the damaged/cracked solar panel. The monitoring is carried out using current sensors which senses current of the individual solar panels and with a help of microcontroller, raspberry Pi 3 and Relay driver it is taken to the load. Raspberry Pi 3 interfaces with mobile application using Wi-Fi technology and updates the status of the solar panel to the users all around the world.
\end{abstract}

Keywords: Solar Cell, Internet of Things (IoT), Crack Identification, Mobile Application, Inspection Technique and Current Sensors.

\section{INTRODUCTION}

\section{History of Solar Cell Crack Detection}

Due to the growing demand of efficient renewable power source, solar panels became very popular as it works by allowing photons, or particles of light (from the sun), to knock electrons free from atoms, generating a flow of electricity. These solar panels are not efficient always as after a long use the efficiency of solar panels may reduce gradually. To identify the cause for the reduction in efficiency many researches were conducted. Finally, it was concluded that the cracks or micro-cracks developed in the solar panel is the main cause for the reduction in efficiency of solar panel. In the early 2000s thermo graphic inspection method was introduced to identify the cracks present in the solar panels. For the detection of surface cracks, a practical way to proceed consists to have the thermal front propagating along the surface, known as lateral surface heating.

This takes advantage of the large thermal resistance at the interface created by the cracks. Another method introduced in the early 2000s was the solar cell crack inspection by image processing. In image processing technique, we first take the image of the solar panel and convert it into a grey scale image, as the grey scale image retains most of the information and does not affect the crack inspection. Due to the dispersed gray values of pixels and the ambiguous characteristic of cracks, inverse operation on histogram equalization should be taken to centralize the distribution of gray value, and then transforming the image to black-white one, which can withstand the cracks.

\section{Need for Solar Cell Crack Detection}

The increasing demand for solar energy has multiplied the need for photovoltaic (PV) arrays. As a major component of the PV array, the demand for solar cells has also increased. Solar cell micro-cracks can occur in different stages of the solar cell lifetime, starting from the manufacturing process, to transportation and installation of the photovoltaic (PV) modules. Due to these cracks the user is forced to replace the entire solar panel which is uneconomical. So we need an improvised technique to detect the crack in the particular cell and replace that cell which will give us longer lifetime of the solar panel and will be economical. The crack detection technique must also help in increasing the efficiency of the solar panel by replacing the cracked solar cell alone. This will enhance the overall output and gives the same performance as of a new solar panel. As there is an abrupt increase in the usage of solar panels in all industrial sectors like automobiles, electronic gadgets etc., the improvised detection method must be able to analyze the damage or crack to the solar panel that occurs easily due to external conditions.

\section{EXISTING TECHNIQUES}

1. Micro-Crack Identification Using Thermal Imaging Quality assurance is of fundamental importance for solar panels. The failure-free operation of the panels is a prerequisite for efficient power generation, long life, and a high return on the investment. To ensure this failure free operation a fast, simple and reliable method to evaluate a 
solar panel's performance is required, both during the production process and after the panel has been installed With a thermal imaging camera, potential problem areas can be detected and repaired before actual problems or failures occur while it is operating and detecting the cracks on the panel where ever the thermal image shows red spots which means (the red spot area has the highest temperature compared to the rest of the area.). This method is used to find many cracks from micro to crystalline cracks.

\section{Limitations Of Thermal Imaging}

Not every thermal imaging camera is suited for solar cell inspection, and there are some rules and guidelines that need to be followed in order to perform efficient inspections and to ensure that you draw correct conclusions. Where thermal imaging technique only helps us to find where the crack is present in the solar panel. Thermal imaging is uneconomical and the thermal cameras costs high and the detection technique is complex.

3. Micro-Crack Identification Using Electroluminescence It is a method for detecting micro-cracks in solar cells using two dimensional matched filters was developed, derived from the electroluminescence intensity profile of typical micro-cracks. This technique shows the image processing steps to obtain a binary map with the location of the micro-cracks. It shows how to automatically estimate the total length of each micro-crack from these maps, and propose a method to identify severe types of micro-cracks, such as parallel, series, and cracks with multiple orientations. With an optimized threshold parameter, the technique detects over $90 \%$ of cracks larger than $3 \mathrm{~cm}$ in length. The method shows great potential for quantifying micro-crack damage after manufacturing or module transportation for the determination of a module quality criterion for cell cracking in photovoltaic modules.

\section{Limitations Of Using Electroluminescence Method}

This process is time consuming as we have to disconnect the whole solar power plant to check which solar panel is defective and it may result in disturbance with the interconnection of solar arrays. It uses complex detection technique which involves emission of light at the bottom of the solar panel to obtain the map of solar panel which shows the crack. Manual monitoring is required for this method. And the equipment for this technique is costly.

\section{Advantages Of Proposed Technique}

- Reduced Time Consumption

- No Need To Shutdown Of Power Plant

- Simple Process

- Economical Technique

- Global Alert Using IoT

- Automated Inspection System Round The Clock

\section{SOLAR CELL CRACK IDENTIFICATION}

\section{Objective}

They are triggered by mechanical and chemical natural factors stressing the panel operating in field, such as hail, snow, sun, wind and severe cold. Further stress factors are geared to the thermal cycles of the cells involving contracting, expanding and flexing metal contacts, solder and wire interconnects. The figure 1 shows the detection of the solar cell crack.

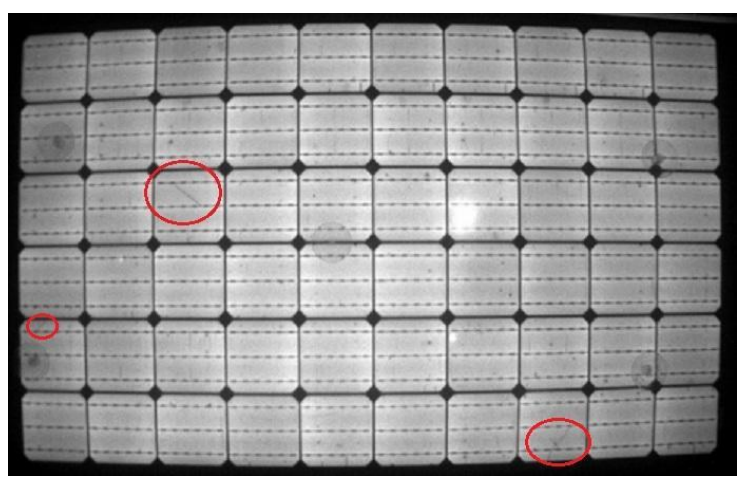

Fig. 1 Solar Cell Crack

2. Existing System

2.1 Thermal Imaging Process

Hand held thermal imaging cameras typically have an uncooled micro bolometer detector that is sensitive in the 8$14 \mu \mathrm{m}$ waveband. However, glass is not transparent in this region. So when solar cells are inspected from the front, a thermal imaging camera sees the heat distribution on the glass surface but only indirectly the thermal performance of the underlying cells.

As a result, the temperature differences that can be measured and seen on the solar panel"s glass surface are small.In order for these differences to be visible, the camera needs a thermal sensitivity of less than $80 \mathrm{k}$ resolution. It should also allow manual adjustment of the level and span function to optimize visual contrast. Photovoltaic modules are generally mounted on highly reflective aluminum framework which shows up as a cold area on a thermal image. This is because it reflects the thermal radiation emitted by the sky. In practice this will mean that a thermal imaging camera will record the framework temperature as being well below $0^{\circ} \mathrm{C}$. As the camera's histogram equalization automatically adapts to the maximum and minimum measured temperatures, many small thermal anomalies will not be immediately visual.

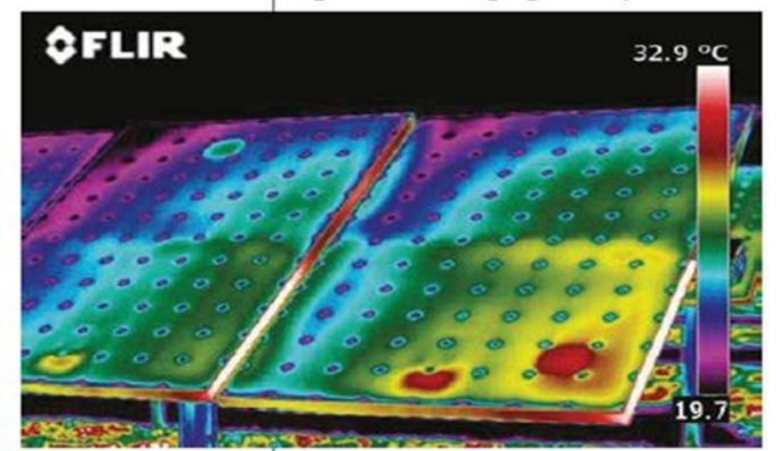

Fig. 2 Thermal Imaging Process on a Solar Cell 
With manual correction of level and span however, clear contrast can be achieved. In this regard, Digital Detail Enhancement (DDE) is a useful function as it automatically optimizes image contrast in high dynamic range scenes. A camera with this feature is therefore particularly well suited to solar panel inspection. Figure 4.2 shows the variation in temperature of the solar cells using thermal imaging process.

\subsection{Electroluminescence Process}

Micro-crack detection in the mono crystalline cell is relatively straightforward because this type of cell is characterized by a uniform background. However, this is not the case for the multi crystalline cell, which contains crystal grains as well as dark areas formed from intrinsic structures like dislocation clusters and grain boundaries. Distinguishing micro-crack pixels from the background (i.e., the multi crystalline grains) is a very challenging procedure because the gray scale values of these two areas are not significantly different. The presence of other defects, such as the dark area, darker grains, and broken fingers complicates the problem. In spite of these difficulties, the identification is still possible because the micro-cracks tend to appear in the form of strong lines with a low intensity and a high gradient. Figure 3 shows an example of Electroluminescence (EL) image of a defected solar cell, and its close-up view of the region containing the micro-crack is displayed in Figure 3.
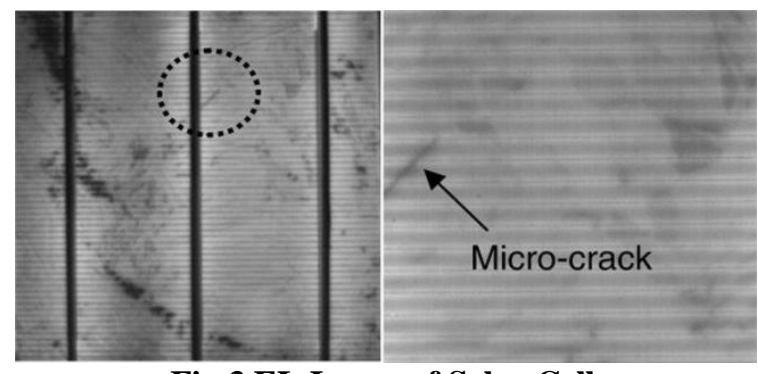

Fig.3 EL Image of Solar Cell

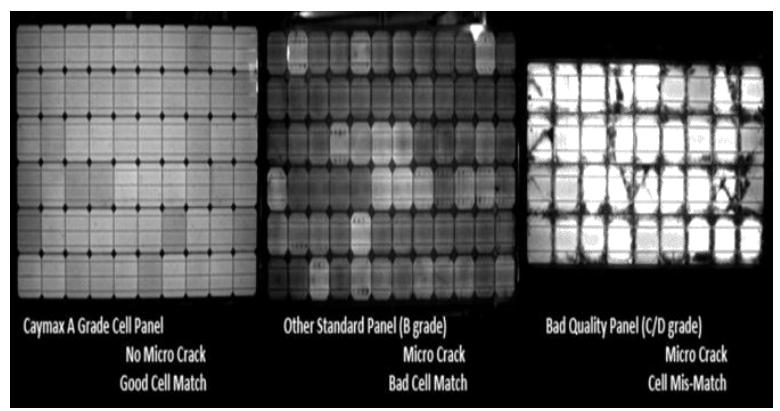

Fig.4 Comparison of Solar Cells under EL Test

3. Disadvantages In Existing Systems

- The entire process is time consuming

- It may lead to temporary shutdown of the entire solar power plant.

- Complex detection techniques.

- Crack/fault alert range is very short.

- Manual monitoring is required.

- System is uneconomical.
May result in disturbance with the interconnection of solar arrays.

\section{Proposed System}

A Photovoltaic module consists of many PV cells wired in parallel to increase current and in series to produce a higher voltage. Figure 4.6 represents a 36 cell modules which are used in the industry for large power production. In practice, huge amount of solar modules are connected in series or parallel for extracting power. Suppose if any cell in a particular module gets damaged, then it will act as a load instead of being a source. So, it is very difficult to identify this type of problem a very vast solar power plant.

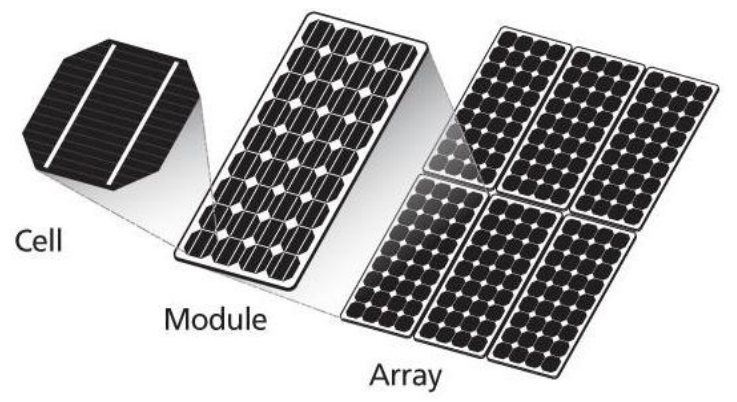

Fig.5 Solar Cells in PV Module

In our proposed technique we use parallel connection in order to obtain different values of current in each panel, to sense the crack in a particular solar panel. Figure 6 shows how solar cells are connected in parallel.

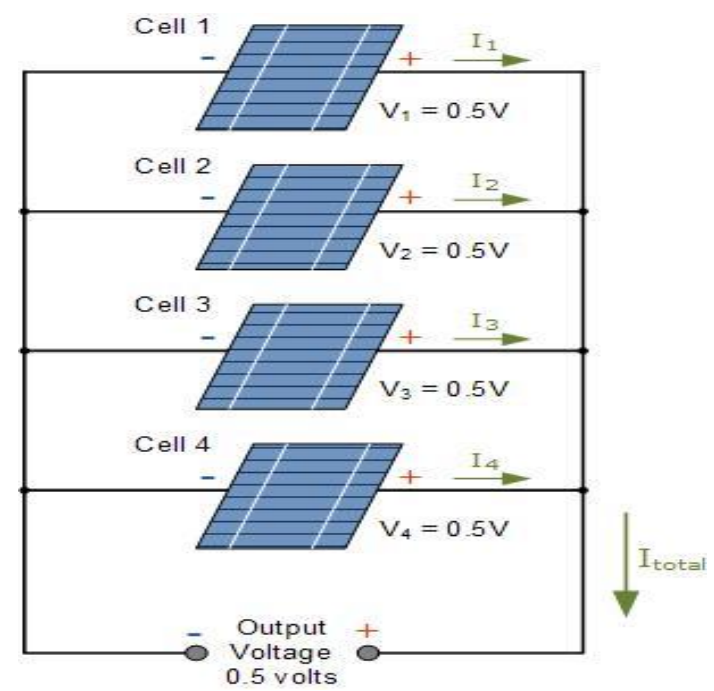

Fig.6 Solar Cells connected in parallel

\section{BLOCK DIAGRAM}

In the proposed system, the output of each panel is interfaced with a current sensor. These current sensors update the output to Raspberry Pi with the help of ADC. If the current level $(270-330 \mathrm{~mA})$ is less than the threshold level in a PV module, then an alert is reported to the control room and/or the energy monitoring application installed in the mobile via internet which is showcased in the figure 7 . 


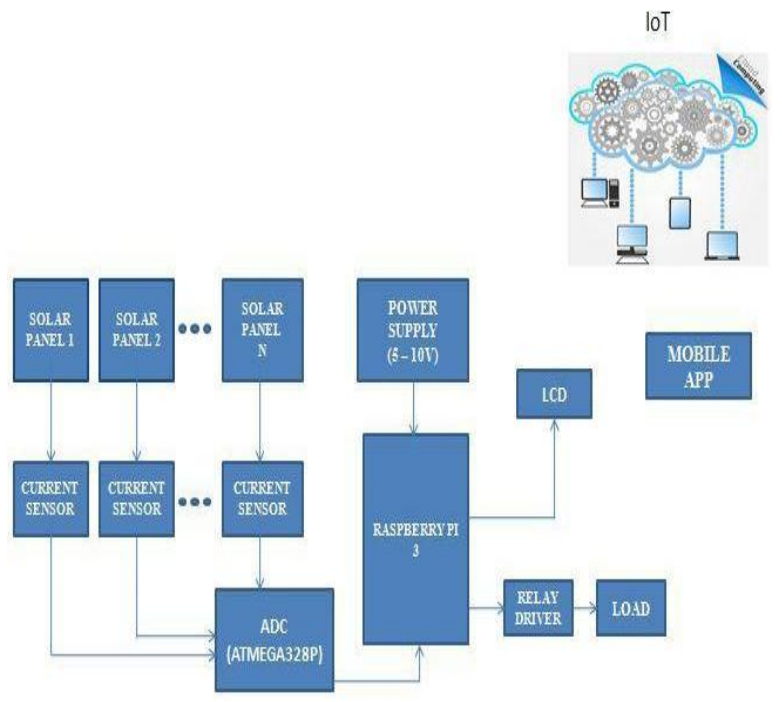

Fig.7 Block Diagram of Proposed System

1. Components

The block diagram as shown in Fig.7 consists of,

1. ADC

2. Sensor

3. $\mathrm{Lpc} 2148$

4. Relay driver

5. Solar

6. LCD

7. Mobile App

\section{Explanation of the Block}

A PV module consists of many PV cells wired in parallel to increase current and in series to produce a higher voltage. 36 cell modules are the industry standard for large power production. The module is encapsulated with tempered glass (or some other transparent material) on the front surface, and with a protective and waterproof material on the back surface. The edges are sealed for weatherproofing, and there is often an aluminum frame holding everything together in a mountable unit. In the back of the module there is a junction box, or wire leads, providing electrical connections. In practice, huge amount of solar modules are connected

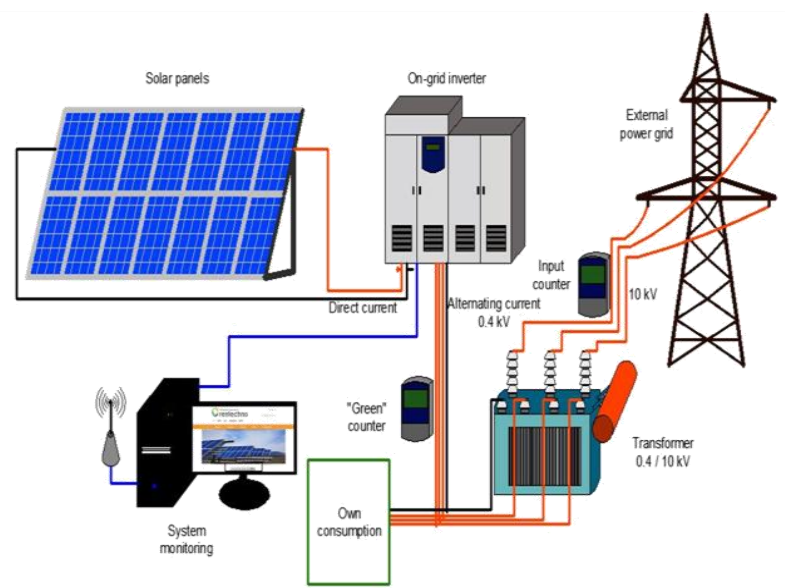

Fig.8 Representation of Solar panel Monitoring in series or parallel for extracting power. Suppose any cells in a particular module got damaged, then it will acts as a load instead of source. It is very difficult to identify this type of problem in a big type of solar plants. To overcome this problem a new crack identification method id proposed. The proposed system has a current sensor and the output of the panel is interfaced with the current sensor. The microcontroller which is linked with the current sensor always watches the current level of the particular module. If the current level is less than the threshold level then the controller disconnect the particular module from the network and inform to the control room. So the wastage of power can be avoided and we can increase the efficiency of the power solar plant.

\section{CONCLUSION}

This automated inspection technique of solar panel with the help of Raspberry Pi microcontroller is more reliable and time saving when compared to other techniques. With this technique the solar panels can be monitored without any manual help reducing human errors. Since the entire process is linked with Internet of Things (IoT), we get update round the clock from anywhere around the globe improving its safety and security features. The scope of the project extends to more than domestic purpose. This project can also be used for large scale industrial purposes, engineering applications and other commercial purposes of solar panel.

\section{FUTURE WORK}

As a future work, the project can be extended with further development such as reducing the size of the entire health monitoring kit with reduction in components used and enhancing the signal coverage area with better connectivity. Through awareness about the solar panel health tracking app, the range of communication can be extended. The inspection technique can be further developed by enhanced circuit design and material design to consume lower energy when compared to the currently available hardware components.

\section{REFERENCES}

[1] E. D. Dunlop and D. Halton, "The performance of crystalline silicon photovoltaic solar modules after 22 years of continuous outdoor exposure" Progress in Photovoltaics 14 (2006), pp. 53-64.

[2] T. Ishii, T. Takashima, and K. Otani, "Long-term performance degradation of various kinds of photovoltaic modules under moderate climatic conditions", Progress in Photovoltaic 19 (2011), pp. 170-179.

[3] M. Vazquez and I. Rey-Stolle, "Photovoltaic module reliability model based on field degradation studies", Progress in Photovoltaics 16 (2008), pp. 419-433.

[4] P. Sanchez-Friera, M. Piliougine, J. Pelaez, J. Carretero, and M. S. de Cardona, "Analysis of degradation mechanisms of crystalline silicon PV modules after 12 years of operation in Southern Europe" Progress in Photovoltaic 19 (2011), pp. 658-666.

[5] M. Köntges, S. Kajari-Schröder, I. Kunze, and U. Jahn, "Crack Statistic of Crystalline Silicon Photovoltaic Modules," in $26^{\text {th }}$ European Photovoltaic Solar Energy Conference and Exhibition, Hamburg, Germany, 2011, pp. 3290 - 3294. 